Commun. Fac Sci. Univ. Ank. Ser. A1 Math. Stat.

Volume 69, Number 1, Pages 629-645(2020)

DOI: $10.31801 /$ cfsuasmas.487296

ISSN 1303-5991 E-ISSN 2618-6470

http://communications.science.ankara.edu.tr/index.php?series=A1

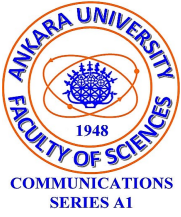

\title{
ON GENERALIZED CHEEGER-GROMOLL METRIC AND
} HARMONICITY

\author{
R. KADA BEN OTMANE, A. ZAGANE, AND M. DJAA
}

\begin{abstract}
In this paper, we introduce the Generalized Cheeger-Gromoll metric on the tangent bundle $T M$, as a natural metric on $T M$. We establish a necessary and sufficient conditions under which a vector field is harmonic with respect to the Generalized Cheeger-Gromoll metric. We also construct some examples of harmonic vector fields.
\end{abstract}

\section{INTRODUCTION}

Consider a smooth map $\phi:\left(M^{m}, g\right) \rightarrow\left(N^{n}, h\right)$ between two Riemannian manifolds, then the energy functional is defined by

$$
E(\phi)=\int_{K} e(\phi) d v_{g}
$$

or over any compact subset $K \subset M$.

$$
e(\phi)=\frac{1}{2} \operatorname{trace}_{g}\left(\phi^{*} h\right)=\frac{1}{2} \operatorname{trace}_{g} h(d \phi, d \phi)
$$

is the energy density of $\phi$.

A map is called harmonic if it is a critical point of the energy functional. For any smooth variation $\left\{\phi_{t}\right\}_{t \in I}$ of $\phi$ with $\phi_{0}=\phi$ and $V=\left.\frac{d}{d t} \phi_{t}\right|_{t=0}$, we have

$$
\left.\frac{d}{d t} E\left(\phi_{t}\right)\right|_{t=0}=-\int_{K} h(\tau(\phi), V) d v_{g}
$$

where

$$
\tau(\phi)=\text { trace }_{g} \nabla d \phi
$$

is the tension field of $\phi$. Then $\phi$ is harmonic if and only if $\tau(\phi)=0$.

One can refer to [14, 15, 22] for background on harmonic maps and 9], 12] for background on generalized harmonic maps.

Received by the editors: November 24, 2018; Accepted: January 14, 2020.

2010 Mathematics Subject Classification. Primary 53A45, 53C20; Secondary 58E20.

Key words and phrases. Horizontal lift, vertical lift, generalized Cheeger-Gromoll metric, harmonic maps.

(C)2020 Ankara University Communications Faculty of Sciences University of Ankara-Series A1 Mathematics and Statistics 
The geometry of the tangent bundle $T M$ equipped with the Sasaki metric has been studied by many authors such as Sasaki [25], K.Yano and S. Ishihara [27], P.Dombrowski [13], A. Salimov, A. Gezer and N. Cengiz [26, 23], etc... The rigidity of Sasaki metric has incited some geometers to construct and study other metrics on $T M$. J. Cheeger and D. Gromoll has introduced the notion of Cheeger-Gromoll metric [5], this metric has been studied also by many authors (see [1], [2], 16], 17], [24, 26]).

The existence and explicit construction of harmonic mappings between two given Riemannian manifolds $(M, g)$ and $(N, h)$ are two of the most fundamental problems of the theory of harmonic mappings. If $M$ is compact $N$ has non positive sectional curvature, then any smooth map from $M$ to $N$ can be deformed into a harmonic map using the heat flow method [Eells and Sampson 1964]. However, there is no general existence theory of harmonic mappings if the target manifold does not satisfy the non positive curvature condition. This fact makes it interesting to find harmonic maps defined by vector fields as a maps from Riemannian manifold $(M, g)$ to its tangent bundle $T M$.

The main idea in this note consists in the modification of the Sasaki metric. First we introduce a natural metric called Generalized Cheeger-Gromoll metric on the tangent bundle $T M$, originally defined by M. Anastasiei [2]. Afterward we establish necessary and sufficient conditions under which a vector field is harmonic with respect to the Generalized Cheeger-Gromoll metric (Theorem 10 and Theorem 11). We also construct some examples of harmonic vector fields and we give a formula for the construction of non trivial examples of vector fields (Theorem 16 and Corollary 17 ). After that we study the harmonicity of the map $\sigma:(M, g) \longrightarrow(T N, \widetilde{h})$ (Theorem 21. Theorem 22 ) and the map $\phi:(T M, \widetilde{g}) \longrightarrow(N, h)$ (Theorem 24. Theorem 25].

1.1. Basic Notion and Definition on $T M$. Let $(M, g)$ be an $m$-dimensional Riemannian manifold and $(T M, \pi, M)$ be its tangent bundle. A local chart $\left(U, x^{i}\right)_{i=\overline{1, m}}$ on $M$ induces a local chart $\left(\pi^{-1}(U), x^{i}, y^{i}\right)_{i=\overline{1, m}}$ on $T M$. Denote by $\Gamma_{i j}^{k}$ the Christoffel symbols of $g$ and by $\nabla$ the Levi-Civita connection of $g$.

We have two complementary distributions on $T M$, the vertical distribution $\mathcal{V}$ and the horizontal distribution $\mathcal{H}$, defined by:

$$
\begin{gathered}
\mathcal{V}_{(x, u)}=\operatorname{ker}\left(d \pi_{(x, u)}\right)=\left\{\left.a^{i} \frac{\partial}{\partial y^{i}}\right|_{(x, u)} ; a^{i} \in \mathbb{R}\right\}, \\
\mathcal{H}_{(x, u)}=\left\{\left.a^{i} \frac{\partial}{\partial x^{i}}\right|_{(x, u)}-\left.a^{i} u^{j} \Gamma_{i j}^{k} \frac{\partial}{\partial y^{k}}\right|_{(x, u)} ; a^{i} \in \mathbb{R}\right\},
\end{gathered}
$$


where $(x, u) \in T M$, such that $T_{(x, u)} T M=\mathcal{H}_{(x, u)} \oplus \mathcal{V}_{(x, u)}$. Let $X=X^{i} \frac{\partial}{\partial x^{i}}$ be a local vector field on $M$. The vertical and the horizontal lifts of $X$ are defined by:

$$
\begin{aligned}
X^{V} & =X^{i} \frac{\partial}{\partial y^{i}} \\
X^{H} & =X^{i} \frac{\delta}{\delta x^{i}}=X^{i}\left\{\frac{\partial}{\partial x^{i}}-y^{j} \Gamma_{i j}^{k} \frac{\partial}{\partial y^{k}}\right\}
\end{aligned}
$$

For consequences, we have $\left(\frac{\partial}{\partial x^{i}}\right)^{H}=\frac{\delta}{\delta x^{i}}$ and $\left(\frac{\partial}{\partial x^{i}}\right)^{V}=\frac{\partial}{\partial y^{i}}$, then $\left(\frac{\delta}{\delta x^{i}}, \frac{\partial}{\partial y^{i}}\right)_{i=\overline{1, m}}$ is a local adapted frame on $T T M$.

If $w=w^{i} \frac{\partial}{\partial x^{i}}+\bar{w}^{j} \frac{\partial}{\partial y^{j}} \in T_{(x, u)} T M$, then its horizontal and vertical parts are defined by

$$
\begin{aligned}
w^{h} & =w^{i} \frac{\partial}{\partial x^{i}}-w^{i} u^{j} \Gamma_{i j}^{k} \frac{\partial}{\partial y^{k}} \in \mathcal{H}_{(x, u)} \\
w^{v} & =\left\{\bar{w}^{j}+w^{i} u^{j} \Gamma_{i j}^{k}\right\} \frac{\partial}{\partial y^{k}} \in \mathcal{V}_{(x, u)}
\end{aligned}
$$

Proposition 1. 27 Let $(M, g)$ be a Riemannian manifold and $R$ its tensor curvature, then for all vector fields $X, Y \in \Gamma(T M)$ and $p=(x, u) \in T M$ we have:

(1) $\left[X^{H}, Y^{H}\right]_{p}=[X, Y]_{p}^{H}-\left(R_{x}(X, Y) u\right)^{V}$,

(2) $\left[X^{H}, Y^{V}\right]_{p}=\left(\nabla_{X} Y\right)_{p}^{V}$,

(3) $\left[X^{V}, Y^{V}\right]_{p}=0$.

\section{Generalized Cheeger-Gromoll metric}

\subsection{Generalized Cheeger-Gromoll metric.}

Definition 2. Let $(M, g)$ be a Riemannian manifold and $\alpha, \beta: \mathbb{R}^{+} \rightarrow \mathbb{R}^{+}, \alpha \neq 0$ are smooth functions. On the tangent bundle $T M$, we define a Generalized CheegerGromoll metric noted $\widetilde{g}$ by:

(1) $\widetilde{g}\left(X^{H}, Y^{H}\right)_{p}=g_{x}(X, Y)$,

(2) $\widetilde{g}\left(X^{H}, Y^{V}\right)_{p}=0$

(3) $\widetilde{g}\left(X^{V}, Y^{V}\right)_{p}=\alpha(r) g(X, Y)+\beta(r) g(X, u) g(Y, u)$,

where $X, Y \in \Gamma(T M), p=(x, u) \in T M$ and $r=g(u, u)$.

For more details see [2].

Remark 3. 1) If $\alpha=1$ and $\beta=0$, then $\widetilde{g}$ is the Sasaki metric [25],

2) If $\alpha=\beta=\frac{1}{r+1}$, then $\widetilde{g}$ is the Cheeger-Gromoll metric [5], [17].

Lemma 4. 1], 16] Let $(M, g)$ be a Riemannian manifold and $f: \mathbb{R} \rightarrow \mathbb{R}$ a smooth function. For all $X, Y \in \Gamma(T M), p=(x, u) \in T M, u=u^{i} \frac{\partial}{\partial x^{i}} \in T_{x} M$ and $r=g(u, u)$, we have: 
(1) $X^{H}(f(r))_{p}=0$,

(2) $X^{V}(f(r))_{p}=2 f^{\prime}(r) g(X, u)_{x}$

(3) $X^{H}(g(Y, u))_{p}=g\left(\nabla_{X} Y, u\right)_{x}$,

(4) $X^{V}(g(Y, u))_{p}=g(X, Y)_{x}$.

Proof. Locally, the statement is a direct consequence of formulas (??) and (5).

Lemma 5. Let $(M, g)$ be a Riemannian manifold and $(T M, \widetilde{g})$ its tangent bundle equipped with the Generalized Cheeger-Gromoll metric, we have:

1) $X^{H} \widetilde{g}\left(Y^{V}, Z^{V}\right)=\widetilde{g}\left(\left(\nabla_{X} Y\right)^{V}, Z^{V}\right)+\widetilde{g}\left(Y^{V},\left(\nabla_{X} Z\right)^{V}\right)$,

2) $X^{V} \widetilde{g}\left(Y^{V}, Z^{V}\right)=2 \alpha^{\prime} g(X, u) g(Y, Z)+2 \beta^{\prime} g(X, u) g(Y, u) g(Z, u)$

$$
+\beta[g(Z, u) g(X, Y)+g(Y, u) g(X, Z)]
$$

for all $X, Y, Z \in \Gamma(T M)$.

Proof. Using Lemma 4, we obtain:

$$
\begin{aligned}
1) X^{H} \widetilde{g}\left(Y^{V}, Z^{V}\right)= & X^{H}[\alpha g(X, Y)+\beta g(X, u) g(Y, u)] \\
= & \left.\alpha X^{H} g(Y, Z)+\beta X^{H}(g(Y, u) g(Z, u))\right] \\
= & \alpha\left[g\left(\nabla_{X} Y, Z\right)+g\left(Y, \nabla_{X} Z\right)\right] \\
& +\beta\left[g\left(\nabla_{X} Y, u\right) g(Z, u)+g(Y, u) g\left(\nabla_{X} Z, u\right)\right] \\
= & \widetilde{g}\left(\left(\nabla_{X} Y\right)^{V}, Z^{V}\right)+\widetilde{g}\left(Y^{V},\left(\nabla_{X} Z\right)^{V}\right) . \\
2) X^{V} \widetilde{g}\left(Y^{V}, Z^{V}\right)= & \left.X^{V}[\alpha g(X, Y)+\beta g(X, u) g(Y, u))\right] \\
= & X^{V}(\alpha) g(Y, Z)+X^{V}(\beta) g(Y, u) g(Z, u)+\beta X^{V}(g(Y, u) g(Z, u)) \\
= & 2 \alpha^{\prime} g(X, u) g(Y, Z)+2 \beta^{\prime} g(X, u) g(Y, u) g(Z, u) \\
& +\beta g(Z, u) g(X, Y)+\beta g(Y, u) g(X, Z) .
\end{aligned}
$$

\subsection{Levi-Civita connection of the Generalized Cheeger-Gromoll metric.}

Lemma 6. Let $(M, g)$ be a Riemannian manifold and $(T M, \widetilde{g})$ its tangent bundle equipped with the Generalized Cheeger-Gromoll metric. If $\nabla$ (resp. $\widetilde{\nabla})$ denote the Levi-Civita connection of $(M, g)$ (resp. $(T M, \widetilde{g})$, then we have:

1) $\widetilde{g}\left(\widetilde{\nabla}_{X^{H}} Y^{H}, Z^{H}\right)=\widetilde{g}\left(\left(\nabla_{X} Y\right)^{H}, Z^{H}\right)$,

2) $\widetilde{g}\left(\widetilde{\nabla}_{X^{H}} Y^{H}, Z^{V}\right)=-\frac{1}{2} \widetilde{g}\left((R(X, Y) u)^{V}, Z^{V}\right)$,

3) $\widetilde{g}\left(\widetilde{\nabla}_{X^{H}} Y^{V}, Z^{H}\right)=\frac{\alpha(r)}{2} \widetilde{g}\left((R(u, Y) X)^{H}, Z^{H}\right)$,

4) $\widetilde{g}\left(\widetilde{\nabla}_{X^{H}} Y^{V}, Z^{V}\right)=\widetilde{g}\left(\left(\nabla_{X} Y\right)^{V}, Z^{V}\right)$,

5) $\widetilde{g}\left(\widetilde{\nabla}_{X^{V}} Y^{H}, Z^{H}\right)=\frac{\alpha(r)}{2} \widetilde{g}\left((R(u, X) Y)^{H}, Z^{H}\right)$, 
6) $\widetilde{g}\left(\widetilde{\nabla}_{X^{V}} Y^{H}, Z^{V}\right)=0$,

7) $\widetilde{g}\left(\widetilde{\nabla}_{X^{V}} Y^{V}, Z^{H}\right)=0$,

8) $\widetilde{g}\left(\widetilde{\nabla}_{X^{v}} Y^{V}, Z^{V}\right)=\widetilde{g}\left(\frac{\alpha^{\prime}}{\alpha}\left[g(X, u) Y^{V}+g(Y, u) X^{V}\right]\right.$

$$
\left.+\left[\frac{\beta-\alpha^{\prime}}{\alpha+r \beta} g(X, Y)+\frac{\alpha \beta^{\prime}-2 \alpha^{\prime} \beta}{\alpha(\alpha+r \beta)} g(X, u) g(Y, u)\right] U^{V}, Z^{V}\right) .
$$

for all $X, Y, U \in \Gamma(T M), U_{x}=u=u^{i} \frac{\partial}{\partial x^{i}} \in T_{x} M$ and $(x, u) \in T M$.

Proof. Using Lemma 4, Lemma 5 and Kozul formula, we obtain:

1) $2 \widetilde{g}\left(\widetilde{\nabla}_{X^{H}} Y^{H}, Z^{H}\right)=X^{H} \widetilde{g}\left(Y^{H}, Z^{H}\right)+Y^{H} \widetilde{g}\left(Z^{H}, X^{H}\right)-Z^{H} \widetilde{g}\left(X^{H}, Y^{H}\right)$

$$
\begin{aligned}
& +\widetilde{g}\left(Z^{H},\left[X^{H}, Y^{H}\right]\right)+\widetilde{g}\left(Y^{H},\left[Z^{H}, X^{H}\right]\right)-\widetilde{g}\left(X^{H},\left[Y^{H}, Z^{H}\right]\right) \\
= & X g(Y, Z)+Y g(Z, X)-Z g(X, Y)+g(Z,[X, Y]) \\
& +g(Y,[Z, X])-g(X,[Y, Z]) \\
= & 2 g\left(\nabla_{X} Y . Z\right) \\
= & 2 \widetilde{g}\left(\left(\nabla_{X} Y\right)^{H}, Z^{H}\right) .
\end{aligned}
$$

2) $2 \widetilde{g}\left(\widetilde{\nabla}_{X^{H}} Y^{H}, Z^{V}\right)=X^{H} \widetilde{g}\left(Y^{H}, Z^{V}\right)+Y^{H} \widetilde{g}\left(Z^{V}, X^{H}\right)-Z^{V} \widetilde{g}\left(X^{H}, Y^{H}\right)$

$$
\begin{aligned}
& +\widetilde{g}\left(Z^{V},\left[X^{H}, Y^{H}\right]\right)+\widetilde{g}\left(Y^{H},\left[Z^{V}, X^{H}\right]\right)-\widetilde{g}\left(X^{H},\left[Y^{H}, Z^{V}\right]\right) \\
= & \widetilde{g}\left(Z^{V},\left[X^{H}, Y^{H}\right]\right) \\
= & -\widetilde{g}\left((R(X, Y) u)^{V}, Z^{V}\right) .
\end{aligned}
$$

3) $2 \widetilde{g}\left(\widetilde{\nabla}_{X^{H}} Y^{V}, Z^{H}\right)=X^{H} \widetilde{g}\left(Y^{V}, Z^{H}\right)+Y^{V} \widetilde{g}\left(Z^{H}, X^{H}\right)-Z^{H} \widetilde{g}\left(X^{H}, Y^{V}\right)$

$$
\begin{aligned}
& +\widetilde{g}\left(Z^{H},\left[X^{H}, Y^{V}\right]\right)+\widetilde{g}\left(Y^{V},\left[Z^{H}, X^{H}\right]\right)-\widetilde{g}\left(X^{H},\left[Y^{V}, Z^{H}\right]\right) \\
= & -\widetilde{g}\left((R(Z, X) u)^{V}, Y^{V}\right) \\
= & -\alpha g(R(Z, X) u, Y)-\beta g(Y, u) g(R(Z, X) u, u)] \\
= & \alpha g(R(u, Y) X, Z) \\
= & \alpha \widetilde{g}\left((R(u, Y) X)^{H}, Z^{H}\right) .
\end{aligned}
$$

4) $2 \widetilde{g}\left(\widetilde{\nabla}_{X^{H}} Y^{V}, Z^{V}\right)=X^{H} \widetilde{g}\left(Y^{V}, Z^{V}\right)+Y^{V} \widetilde{g}\left(Z^{V}, X^{H}\right)-Z^{V} \widetilde{g}\left(X^{H}, Y^{V}\right)$

$$
\begin{aligned}
& +\widetilde{g}\left(Z^{V},\left[X^{H}, Y^{V}\right]\right)+\widetilde{g}\left(Y^{V},\left[Z^{V}, X^{H}\right]\right)-\widetilde{g}\left(X^{H},\left[Y^{V}, Z^{V}\right]\right) \\
= & X^{H} \widetilde{g}\left(Y^{V}, Z^{V}\right)+\widetilde{g}\left(Z^{V},\left[X^{H}, Y^{V}\right]\right)+\widetilde{g}\left(Y^{V},\left[Z^{V}, X^{H}\right]\right) \\
= & \widetilde{g}\left(\left(\nabla_{X} Y\right)^{V}, Z^{V}\right)+\widetilde{g}\left(Y^{V},\left(\nabla_{X} Z\right)^{V}\right) \\
& +\widetilde{g}\left(Z^{V},\left(\nabla_{X} Y\right)^{V}\right)-\widetilde{g}\left(Y^{V},\left(\nabla_{X} Z\right)^{V}\right) \\
= & 2 \widetilde{g}\left(\left(\nabla_{X} Y\right)^{V}, Z^{V}\right) .
\end{aligned}
$$


The other formulas are obtained by a similar calculation.

Theorem 7. [16, 21] Let $(M, g)$ be a Riemannian manifold and $(T M, \widetilde{g})$ its tangent bundle equipped with the Generalized Cheeger-Gromoll metric. If $\nabla$ (resp. $\widetilde{\nabla})$ denote the Levi-Civita connection of $(M, g)($ resp. $(T M, \widetilde{g}))$, then we have:

1) $\left(\widetilde{\nabla}_{X^{H}} Y^{H}\right)_{p}=\left(\nabla_{X} Y\right)_{p}^{H}-\frac{1}{2}\left(R_{x}(X, Y) u\right)^{V}$,

2) $\left(\widetilde{\nabla}_{X^{H}} Y^{V}\right)_{p}=\left(\nabla_{X} Y\right)_{p}^{V}+\frac{\alpha}{2}\left(R_{x}(u, Y) X\right)^{H}$,

3) $\left(\widetilde{\nabla}_{X^{V}} Y^{H}\right)_{p}=\frac{\alpha}{2}\left(R_{x}(u, X) Y\right)^{H}$,

4) $\left(\widetilde{\nabla}_{X^{V}} Y^{V}\right)_{p}=\frac{\alpha^{\prime}}{\alpha}\left[g_{x}(X, u) Y_{p}^{V}+g_{x}(Y, u) X_{p}^{V}\right]$ $+\left[\frac{\beta-\alpha^{\prime}}{\alpha+r \beta} g_{x}(X, Y)+\frac{\alpha \beta^{\prime}-2 \alpha^{\prime} \beta}{\alpha(\alpha+r \beta)} g_{x}(X, u) g_{x}(Y, u)\right] U_{p}^{V}$.

for all $X, Y, U \in \Gamma(T M), U_{x}=u=u^{i} \frac{\partial}{\partial x^{i}} \in T_{x} M$ and $p=(x, u) \in T M$. where $R$ denote the curvature tensor of $(M, g)$.

Proof. The statement is a direct consequence of Lemma 6 .

\section{Generalized Cheeger-Gromoll metric and Harmonicity}

\subsection{Harmonicity of a vector field $X:(M, g) \longrightarrow(T M, \widetilde{g})$.}

Lemma 8. Let $(M, g)$ be a Riemannian manifold. If $X, Y \in \Gamma(T M)$ are vector fields on $M$ and $(x, u) \in T M$ such that $Y_{x}=u$, then we have:

$$
d_{x} Y\left(X_{x}\right)=X_{(x, u)}^{H}+\left(\nabla_{X} Y\right)_{(x, u)}^{V} .
$$

Proof. Let $\left(U, x^{i}\right)$ be a local chart on $M$ in $x \in M$ and $\left.\pi^{(-1)}(U), x^{i}, y^{j}\right)$ be the induced chart on $T M$, if $X_{x}=\left.X^{i}(x) \frac{\partial}{\partial x^{i}}\right|_{x}$ and $Y_{x}=\left.Y^{i}(x) \frac{\partial}{\partial x^{i}}\right|_{x}=u$, then

$$
d_{x} Y\left(X_{x}\right)=\left.X^{i}(x) \frac{\partial}{\partial x^{i}}\right|_{(x, u)}+\left.X^{i}(x) \frac{\partial Y^{k}}{\partial x^{i}}(x) \frac{\partial}{\partial y^{k}}\right|_{(x, u)},
$$

thus the horizontal part is given by:

$$
\begin{aligned}
\left(d_{x} Y\left(X_{x}\right)\right)^{h} & =\left.X^{i}(x) \frac{\partial}{\partial x^{i}}\right|_{(x, u)}-\left.X^{i}(x) Y^{j}(x) \Gamma_{i j}^{k}(x) \frac{\partial}{\partial y^{k}}\right|_{(x, u)} \\
& =X_{(x, u)}^{H},
\end{aligned}
$$

and the vertical part is given by:

$$
\begin{aligned}
\left(d_{x} Y\left(X_{x}\right)\right)^{v} & =\left.\left\{X^{j}(x) \frac{\partial Y^{k}}{\partial x^{i}}(x)+X^{i}(x) Y^{j}(x) \Gamma_{i j}^{k}(x)\right\} \frac{\partial}{\partial y^{k}}\right|_{(x, u)} \\
& =\left(\nabla_{X} Y\right)_{(x, u)}^{V} .
\end{aligned}
$$


Lemma 9. Let $\left(M^{m}, g\right)$ be a Riemannian m-dimensional manifold and $(T M, \widetilde{g})$ its tangent bundle equipped with the Generalized Cheeger-Gromoll metric. If $X \in$ $\Gamma(T M)$, then the energy density associated to $X$ is given by:

$$
e(X)=\frac{m}{2}+\frac{1}{2} \text { trace }_{g}\left[\alpha g(\nabla X, \nabla X)+\beta g(\nabla X, u)^{2}\right] .
$$

Proof. Let $X \in \Gamma(T M)$ and $\left(E_{1}, \cdots, E_{m}\right)$ be a local orthonormal frame on $M$, then:

$$
e(X)=\frac{1}{2} \sum_{i=1}^{m} \widetilde{g}\left(d X\left(E_{i}\right), d X\left(E_{i}\right)\right)
$$

Using Lemma 8, we obtain:

$$
\begin{aligned}
e(X) & =\frac{1}{2} \sum_{i=1}^{m} \widetilde{g}\left(E_{i}^{H}+\left(\nabla_{E_{i}} X\right)^{V}, E_{i}^{H}+\left(\nabla_{E_{i}} X\right)^{V}\right) \\
& \left.=\frac{1}{2} \sum_{i=1}^{m}\left[\widetilde{g}\left(E_{i}^{H}, E_{i}^{H}\right)+\widetilde{g}\left(\left(\nabla_{E_{i}} X\right)^{V},\left(\nabla_{E_{i}} X\right)^{V}\right)\right)\right] \\
& =\frac{1}{2} \sum_{i=1}^{m}\left[g\left(E_{i}, E_{i}\right)+\alpha g\left(\nabla_{E_{i}} X, \nabla_{E_{i}} X\right)+\beta g\left(\nabla_{E_{i}} X, u\right)^{2}\right] \\
& =\frac{m}{2}+\frac{1}{2} \text { trace }_{g}\left[\alpha g(\nabla X, \nabla X)+\beta g(\nabla X, u)^{2}\right]
\end{aligned}
$$

Theorem 10. Let $\left(M^{m}, g\right)$ be a Riemannian m-dimensional manifold and $(T M, \widetilde{g})$ its tangent bundle equipped with the Generalized Cheeger-Gromoll metric. If $X \in$ $\Gamma(T M)$, then the tension field associated to $X$ is given by:

$$
\tau(X)=\left[\operatorname{trace}_{g}(\alpha R(X, \nabla X) *)\right]^{H}+\left[\operatorname{trace}_{g} A(X)\right]^{V} .
$$

where $A(X)$ is a bilinear map defined by:

$$
\begin{aligned}
A(X)= & \nabla^{2} X+\frac{2 \alpha^{\prime}}{\alpha} g(\nabla X, X) \nabla X+\left[\frac{\beta-\alpha^{\prime}}{\alpha+\|X\|^{2} \beta} g(\nabla X, \nabla X)\right. \\
& \left.+\frac{\alpha \beta^{\prime}-2 \alpha^{\prime} \beta}{\alpha\left(\alpha+\|X\|^{2} \beta\right)} g(\nabla X, X)^{2}\right] X,
\end{aligned}
$$

and $\|X\|^{2}=g(X, X)$.

Proof. Let $x \in M$ and $\left\{E_{i}\right\}_{i=1, m}$ be a local orthonormal frame on $M$ such that $\left(\nabla_{E_{i}}^{M} E_{i}\right)_{x}=0$ and $X_{x}=u$, then:

$$
\tau(X)_{x}=\sum_{i=1}^{m}\left\{\nabla_{E_{i}}^{X} d X\left(E_{i}\right)-d X\left(\nabla_{E_{i}}^{M} E_{i}\right)\right\}_{x}
$$




$$
\begin{aligned}
& =\sum_{i=1}^{m}\left\{\widetilde{\nabla}_{d X\left(E_{i}\right)} d X\left(E_{i}\right)\right\}_{(x, u)} \\
& =\sum_{i=1}^{m}\left\{\widetilde{\nabla}_{\left[E_{i}^{H}+\left(\nabla_{E_{i}} X\right)^{V}\right]}\left[E_{i}^{H}+\left(\nabla_{E_{i}} X\right)^{V}\right]\right\}_{(x, u)} \\
& =\sum_{i=1}^{m}\left\{\widetilde{\nabla}_{E_{i}^{H}} E_{i}^{H}+\widetilde{\nabla}_{E_{i}^{H}}\left(\nabla_{E_{i}} X\right)^{V}+\widetilde{\nabla}_{\left(\nabla_{E_{i}} X\right)^{V}}\left(E_{i}\right)^{H}+\widetilde{\nabla}_{\left(\nabla_{E_{i}} X\right)^{V}}\left(\nabla_{E_{i}} X\right)^{V}\right\}_{(x, u)},
\end{aligned}
$$

Using Theorem 7 we obtain:

$$
\begin{aligned}
\tau(X)= & \sum_{i=1}^{m}\left[\left(\nabla_{E_{i}} E_{i}\right)^{H}-\frac{1}{2}\left(R\left(E_{i}, E_{i}\right) X\right)^{V}+\left(\nabla_{E_{i}} \nabla_{E_{i}} X\right)^{V}+\frac{\alpha}{2}\left(R\left(X, \nabla_{E_{i}} X\right) E_{i}\right)^{H}\right. \\
& +\frac{\alpha}{2}\left(R\left(X, \nabla_{E_{i}} X\right) E_{i}\right)^{H}+\frac{\alpha^{\prime}}{\alpha}\left[g\left(\nabla_{E_{i}} X, X\right)\left(\nabla_{E_{i}} X\right)^{V}+g\left(\nabla_{E_{i}} X, X\right)\left(\nabla_{E_{i}} X\right)^{V}\right] \\
& \left.+\left[\frac{\beta-\alpha^{\prime}}{\alpha+\|X\|^{2} \beta} g\left(\nabla_{E_{i}} X, \nabla_{E_{i}} X\right)+\frac{\alpha \beta^{\prime}-2 \alpha^{\prime} \beta}{\alpha\left(\alpha+\|X\|^{2} \beta\right)} g\left(\nabla_{E_{i}} X, X\right) g\left(\nabla_{E_{i}} X, X\right)\right] X^{V}\right] \\
= & \sum_{i=1}^{m}\left[\alpha\left(R\left(X, \nabla_{E_{i}} X\right) E_{i}\right)^{H}+\left(\nabla_{E_{i}} \nabla_{E_{i}} X\right)^{V}+\frac{2 \alpha^{\prime}}{\alpha} g\left(\nabla_{E_{i}} X, X\right)\left(\nabla_{E_{i}} X\right)^{V}\right. \\
& \left.+\left[\frac{\beta-\alpha^{\prime}}{\alpha+\|X\|^{2} \beta} g\left(\nabla_{E_{i}} X, \nabla_{E_{i}} X\right)+\frac{\alpha \beta^{\prime}-2 \alpha^{\prime} \beta}{\alpha\left(\alpha+\|X\|^{2} \beta\right)} g\left(\nabla_{E_{i}} X, X\right)^{2}\right] X^{V}\right] \\
= & {[\operatorname{trace} g(\alpha R(X, \nabla X) *)]^{H}+\left[\operatorname { t r a c e } _ { g } \left(\nabla^{2} X+\frac{2 \alpha^{\prime}}{\alpha} g(\nabla X, X) \nabla X\right.\right.} \\
& \left.\left.+\left[\frac{\beta-\alpha^{\prime}}{\alpha+\|X\|^{2} \beta} g(\nabla X, \nabla X)+\frac{\alpha \beta^{\prime}-2 \alpha^{\prime} \beta}{\alpha\left(\alpha+\|X\|^{2} \beta\right)} g(\nabla X, X)^{2}\right] X\right)\right]^{V} .
\end{aligned}
$$

Theorem 11. Let $\left(M^{m}, g\right)$ be a Riemannian m-dimensional manifold and $(T M, \widetilde{g})$ its tangent bundle equipped with the Generalized Cheeger-Gromoll metric. If $X \in$ $\Gamma(T M)$, then $X$ is harmonic if and only the following conditions are verified

$$
\text { trace }_{g}(R(X, \nabla X) *)=0
$$

and

$$
\begin{aligned}
\operatorname{trace}_{g}( & \nabla^{2} X+\frac{2 \alpha^{\prime}}{\alpha} g(\nabla X, X) \nabla X \\
& \left.+\left[\frac{\beta-\alpha^{\prime}}{\alpha+\|X\|^{2} \beta} g(\nabla X, \nabla X)+\frac{\alpha \beta^{\prime}-2 \alpha^{\prime} \beta}{\alpha\left(\alpha+\|X\|^{2} \beta\right)} g(\nabla X, X)^{2}\right] X\right)=0
\end{aligned}
$$

Proof. The statement is a direct consequence of Theorem 10 
Corollary 12. Let $\left(M^{m}, g\right)$ be a Riemannian m-dimensional manifold and $(T M, \widetilde{g})$ its tangent bundle equipped with the Generalized Cheeger-Gromoll metric. If $X \in$ $\Gamma(T M)$ is a parallel (i.e $\nabla X=0$ ), then $X$ is harmonic.

Theorem 13. Let $\left(M^{m}, g\right)$ be a Riemannian compact m-dimensional manifold and $(T M, \widetilde{g})$ its tangent bundle equipped with the Generalized Cheeger-Gromoll metric. If $X \in \Gamma(T M)$, then $X$ is harmonic if and only if $X$ is parallel (i.e $\nabla X=0)$.

Proof. If $X$ is parallel from Corollary 12 , we deduce that $X$ is harmonic vector field.

Inversely, let $\varphi_{t}$ be a compactly supported variation of $X$ defined by:

$$
\begin{aligned}
\varphi: \mathbb{R} \times M & \longrightarrow T_{x} M \\
(t, x) & \longmapsto \varphi(t, x)=\varphi_{t}(x)=(t+1) X_{x}
\end{aligned}
$$

From Lemma 9 we have:

$$
\begin{gathered}
e\left(\varphi_{t}\right)=\frac{m}{2}+\frac{(t+1)^{2}}{2} \operatorname{trace}_{g}\left[\alpha g(\nabla X, \nabla X)+\beta g(\nabla X, X)^{2}\right] \\
E\left(\varphi_{t}\right)=\frac{m}{2} \operatorname{Vol}(M)+\frac{(t+1)^{2}}{2} \int_{M} \operatorname{trace}_{g}\left[\alpha g(\nabla X, \nabla X)+\beta g(\nabla X, X)^{2}\right] d v_{g}
\end{gathered}
$$

If $X$ is a critical point of the energy functional, then we have :

$$
\begin{aligned}
0 & =\left.\frac{\partial}{\partial t} E\left(\varphi_{t}\right)\right|_{t=0} \\
& =\frac{\partial}{\partial t}\left[\frac{m}{2} \operatorname{Vol}(M)+\frac{(t+1)^{2}}{2} \int_{M} \operatorname{trace}_{g}\left[\alpha g(\nabla X, \nabla X)+\beta g(\nabla X, X)^{2}\right] d v_{g}\right]_{t=0} \\
& =\int_{M} \operatorname{trace}_{g}\left[\alpha g(\nabla X, \nabla X)+\beta g(\nabla X, X)^{2}\right] d v_{g}
\end{aligned}
$$

then $g(\nabla X, \nabla X)+g(\nabla X, X)^{2}=0$, hence $\nabla X=0$.

Example 14. Let $\mathbb{R}^{n}$ equipped with the canonical metric (flat manifold and non compact) and the vector field:

$$
\begin{aligned}
X: \mathbb{R}^{n} & \longrightarrow T \mathbb{R}^{n} \\
x=\left(x_{1}, \cdots, x_{n}\right) & \longmapsto X_{x}=\left(X_{x}^{1}, \cdots, X_{x}^{n}\right)
\end{aligned}
$$

we have:

$$
\tau(X)=\sum_{i=1}^{n}\left(\frac{\partial^{2} X^{1}}{\partial x_{i}^{2}}, \ldots, \frac{\partial^{2} X^{n}}{\partial x_{i}^{2}}\right)
$$

1) If $X$ is constant, then $X$ is harmonic.

2) If $X^{i}=a_{i} x_{i}$ and $a_{i} \neq 0$, then $X$ is harmonic $(\tau(X)=0)$ but

$$
\nabla X=\sum_{i} a_{i} \frac{\partial}{\partial x_{i}} \otimes d x_{i} \neq 0
$$


indeed

$$
\nabla X\left(\partial x_{j}\right)=\nabla_{\partial x_{j}} X=\sum_{i} a_{i} \nabla_{\partial x_{j}}\left(x_{i} \frac{\partial}{\partial x_{i}}\right)=\sum_{i} a_{i} \delta_{i j} \frac{\partial}{\partial x_{i}}=a_{j} \frac{\partial}{\partial x_{j}} .
$$

Example 15. Let $\mathbb{S}^{1}$ equipped with the metric:

$$
g_{\mathbb{S}^{1}}=\frac{4}{\left(1+x^{2}\right)^{2}} d x^{2}
$$

as $\mathbb{S}^{1}$ is compact then. The vector field $X=a(x) \frac{\partial}{\partial x}, a \in \mathcal{C}^{\infty}\left(\mathbb{S}^{1}\right)$ is harmonic if and only if $X$ is parallel, i.e

$$
\begin{aligned}
\nabla X=0 & \Leftrightarrow \nabla_{\frac{\partial}{\partial x}} a \frac{\partial}{\partial x}=0 \\
& \Leftrightarrow \frac{\partial a}{\partial x}+a \Gamma=0 \\
& \Leftrightarrow \frac{\partial a}{\partial x}-\frac{2 x}{1+x^{2}} a=0 \\
& \Leftrightarrow a(x)=k\left(1+x^{2}\right), k \in \mathbb{R} \\
& \Leftrightarrow X=k\left(1+x^{2}\right) \frac{\partial}{\partial x}, k \in \mathbb{R}
\end{aligned}
$$

Theorem 16. Let $\left(\mathbb{R}^{m}, g_{0}\right)$ the real euclidean space and $\left(T \mathbb{R}^{m}, \widetilde{g_{0}}\right)$ its tangent bundle equipped with the Generalized Cheeger-Gromoll metric. If $X=\left(X^{1}, \cdots, X^{m}\right) \in$ $\Gamma\left(T \mathbb{R}^{m}\right)$, then $X$ is harmonic if and only if $X$ verifies the following system of equations

$$
\begin{aligned}
\sum_{i=1}^{m} \frac{\partial^{2} X^{k}}{\partial\left(x^{i}\right)^{2}}+\sum_{i, j=1}^{m} & \left(\frac{2 \alpha^{\prime}}{\alpha} X^{j} \frac{\partial X^{j}}{\partial x^{i}} \frac{\partial X^{k}}{\partial x^{i}}+\frac{\beta-\alpha^{\prime}}{\alpha+\|X\|^{2} \beta} X^{k}\left(\frac{\partial X^{j}}{\partial x^{i}}\right)^{2}\right) \\
& +\frac{\alpha \beta^{\prime}-2 \alpha^{\prime} \beta}{\alpha\left(\alpha+\|X\|^{2} \beta\right)} X^{k} \sum_{i=1}^{m}\left(\sum_{j=1}^{m} X^{j} \frac{\partial X^{j}}{\partial x^{i}}\right)^{2}=0 .
\end{aligned}
$$

for all $k=\overline{1, m}$.

Proof. Let $\left\{\frac{\partial}{\partial x^{i}}\right\}_{i=1, m}$ be a canonical frame on $\mathbb{R}^{m}$. Using Theorem 11, we have: $\tau(X)=0$ equivalent the following equations (??) and (??) are verified. As $\mathbb{R}^{m}$ is flat, then the equation (??) is obvious.

Hence,

$$
\begin{aligned}
& \tau(X)=0 \Leftrightarrow(? ?) \\
& \Leftrightarrow \text { trace }_{g}\left[\nabla^{2} X+\frac{2 \alpha^{\prime}}{\alpha} g(\nabla X, X) \nabla X\right. \\
& \left.+\left[\frac{\beta-\alpha^{\prime}}{\alpha+\|X\|^{2} \beta} g(\nabla X, \nabla X)+\frac{\alpha \beta^{\prime}-2 \alpha^{\prime} \beta}{\alpha\left(\alpha+\|X\|^{2} \beta\right)} g(\nabla X, X)^{2}\right] X\right]=0
\end{aligned}
$$




$$
\begin{gathered}
\sum_{i=1}^{m}\left[\nabla_{\frac{\partial}{\partial x^{i}}} \nabla_{\frac{\partial}{\partial x^{i}}} X+\frac{2 \alpha^{\prime}}{\alpha} g\left(\nabla_{\frac{\partial}{\partial x^{i}}} X, X\right)\left(\nabla_{\frac{\partial}{\partial x^{i}}} X\right)\right. \\
\left.+\left[\frac{\beta-\alpha^{\prime}}{\alpha+\|X\|^{2} \beta} g\left(\nabla_{\frac{\partial}{\partial x^{i}}} X, \nabla_{\frac{\partial}{\partial x^{i}}} X\right)+\frac{\alpha \beta^{\prime}-2 \alpha^{\prime} \beta}{\alpha\left(\alpha+\|X\|^{2} \beta\right)} g\left(\nabla_{\frac{\partial}{\partial x^{i}}} X, X\right)^{2}\right] X\right]=0 \\
\Leftrightarrow \sum_{i=1}^{m}\left[\sum_{k=1}^{m}\left(\frac{\partial^{2} X^{k}}{\partial\left(x^{i}\right)^{2}} \frac{\partial}{\partial x^{k}}\right)+\frac{2 \alpha^{\prime}}{\alpha} \sum_{j=1}^{m}\left(\frac{\partial X^{j}}{\partial x^{i}} X^{j}\right) \sum_{k=1}^{m}\left(\frac{\partial X^{k}}{\partial x^{i}} \frac{\partial}{\partial x^{k}}\right)\right. \\
\left.+\left[\frac{\beta-\alpha^{\prime}}{\alpha+\|X\|^{2} \beta} \sum_{j=1}^{m}\left(\frac{\partial X^{j}}{\partial x^{i}}\right)^{2}+\frac{\alpha \beta^{\prime}-2 \alpha^{\prime} \beta}{\alpha\left(\alpha+\|X\|^{2} \beta\right)}\left(\sum_{j=1}^{m} X^{j} \frac{\partial X^{j}}{\partial x^{i}}\right)^{2}\right] \sum_{i=1}^{k}\left(X^{k} \frac{\partial}{\partial x^{k}}\right)\right]=0 \\
\Leftrightarrow \sum_{i=1}^{m} \frac{\partial^{2} X^{k}}{\partial\left(x^{i}\right)^{2}}+\sum_{i, j=1}^{m}\left(\frac{2 \alpha^{\prime}}{\alpha} X^{j} \frac{\partial X^{j}}{\partial x^{i}} \frac{\partial X^{k}}{\partial x^{i}}+\frac{\beta-\alpha^{\prime}}{\alpha+\|X\|^{2} \beta} X^{k}\left(\frac{\partial X^{j}}{\partial x^{i}}\right)^{2}\right) \\
+\frac{\alpha \beta^{\prime}-2 \alpha^{\prime} \beta}{\alpha\left(\alpha+\|X\|^{2} \beta\right)} X^{k} \sum_{i=1}^{m}\left(\sum_{j=1}^{m} X^{j} \frac{\partial X^{j}}{\partial x^{i}}\right)^{2}=0 .
\end{gathered}
$$

for all $k=\overline{1, m}$.

Corollary 17. Let $\left(\mathbb{R}^{m}, g_{0}\right)$ the real euclidean space and $\left(T \mathbb{R}^{m}, \widetilde{g_{0}}\right)$ its tangent bundle equipped with the Generalized Cheeger-Gromoll metric and $X=\left(X^{1}, \cdots, X^{m}\right) \in$ $\Gamma\left(T \mathbb{R}^{m}\right)$. If $\alpha$ and $\beta$ are constant functions, then $X$ is a harmonic if and only if for all $k=\overline{1, m}$ :

$$
\sum_{i=1}^{m} \frac{\partial^{2} X^{k}}{\partial\left(x^{i}\right)^{2}}+\frac{\beta}{\alpha+\|X\|^{2} \beta} X^{k} \sum_{i, j=1}^{m}\left(\frac{\partial X^{j}}{\partial x^{i}}\right)^{2}=0 .
$$

Remark 18. Using Corollary 17, we can construct many examples of non trivial harmonic vector fields.

Example 19. If $\mathbb{R}^{n}$ is endowed with the canonical metric and $T \mathbb{R}^{m}$ its tangent bundle equipped with the Generalized Cheeger-Gromoll metric. From corollary 17 . we deduce that:

1) If $X=\left(y\left(x_{1}\right), 0, \cdots, 0\right) \in \Gamma\left(T \mathbb{R}^{m}\right)$ is a harmonic vector field if and only the function $y$ is solution of differential equation:

$$
y^{\prime \prime}+\frac{\beta y^{\prime} y}{\alpha+\beta y^{2}} y^{\prime}=0 .
$$

2) If $X=\left(y\left(x_{1}, x_{2}\right), 0, \cdots, 0\right) \in \Gamma\left(T \mathbb{R}^{m}\right)$ is a harmonic vector field if and only the function $y$ is the solution of the partial derivative equation:

$$
\frac{\partial^{2} y}{\partial x_{1}^{2}}+\frac{\partial^{2} y}{\partial x_{2}^{2}}+\frac{\beta y}{\alpha+\beta y^{2}}\left(\frac{\partial y}{\partial x_{1}}+\frac{\partial y}{\partial x_{2}}\right)=0 .
$$

where $\alpha, \beta \in \mathbb{R}^{+}$. 
3.2. Harmonicity of the map $\sigma:(M, g) \longrightarrow(T N, \widetilde{h})$.

Lemma 20. Let $\left(M^{m}, g\right),\left(N^{n}, h\right)$ two Riemannian manifolds and $\left.f: N \rightarrow\right] 0,+\infty[$ a smooth function. Let $(T N, \widetilde{h})$ the tangent bundle of $N$ equipped with the Generalized Cheeger-Gromoll metric. If

$$
\begin{aligned}
\sigma:(M, g) & \longrightarrow(T N, \widetilde{h}) \\
x & \longmapsto(\varphi(x), v)
\end{aligned}
$$

a map, such that $\varphi=\pi_{T N} \circ \sigma$ and $v=Y_{\varphi(x)} \in T_{\varphi(x)} N$ where $Y \in \Gamma(T N)$, $\pi_{T N}: T N \rightarrow N$ is the canonical projection, then:

$$
d \sigma(X)=(d \varphi(X))^{H}+\left(\nabla_{X}^{\varphi} \sigma\right)^{V}
$$

for all $X \in \Gamma(T M)$.

Proof. Using Lemma 8 , we obtain:

$$
\begin{aligned}
d_{x} \sigma\left(X_{x}\right) & =d_{x}(Y \circ \varphi)\left(X_{x}\right) \\
& =d_{\varphi(x)} Y\left(d \varphi\left(X_{x}\right)\right) \\
& =(d \varphi(X))_{(\varphi(x), v)}^{H}+\left(\nabla_{d \varphi(X)} Y\right)_{(\varphi(x), v)}^{V} \\
& =(d \varphi(X))_{(\varphi(x), v)}^{H}+\left(\nabla_{X}^{\varphi} \sigma\right)_{(\varphi(x), v)}^{V}
\end{aligned}
$$

where $Y_{\varphi(x)}=v \in T_{\varphi(x)} N$

Theorem 21. Let $\left(M^{m}, g\right),\left(N^{n}, h\right)$ two Riemannian manifolds and $f: N \rightarrow$ ] $0,+\infty[$ a smooth function. Let $(T N, \widetilde{h})$ the tangent bundle of $N$ equipped with the Generalized Cheeger-Gromoll metric. The tension field of the map

$$
\begin{aligned}
\sigma:(M, g) & \longrightarrow\left(T N, h^{f}\right) \\
x & \longmapsto(\varphi(x), v)
\end{aligned}
$$

such that $\varphi=\pi_{T N} \circ \sigma$, is given by:

$$
\tau(\sigma)=\left[\tau(\varphi)+\operatorname{trace}_{g}\left(\alpha R^{N}\left(\sigma, \nabla^{\varphi} \sigma\right) d \varphi(*)\right)\right]^{H}+\left[\operatorname{trace}_{g} A(\sigma)\right]^{V}
$$

where $A(\sigma)$ is a bilinear map defined by:

$$
\begin{aligned}
A(\sigma)= & \left(\nabla^{\varphi}\right)^{2} \sigma+\frac{2 \alpha^{\prime}}{\alpha} h\left(\nabla^{\varphi} \sigma, \sigma\right) \nabla^{\varphi} \sigma \\
& +\left[\frac{\beta-\alpha^{\prime}}{\alpha+\|\sigma\|^{2} \beta} h\left(\nabla^{\varphi} \sigma, \nabla^{\varphi} \sigma\right)+\frac{\alpha \beta^{\prime}-2 \alpha^{\prime} \beta}{\alpha\left(\alpha+\|\sigma\|^{2} \beta\right)} h\left(\nabla^{\varphi} \sigma, \sigma\right)^{2}\right] \sigma
\end{aligned}
$$

and $\|\sigma\|^{2}=h(\sigma, \sigma)=r$.

Proof. Let $x \in M$ and $\left\{E_{i}\right\}_{i=\overline{1, m}}$ be a local orthonormal frame on $M$ such that $\left(\nabla_{E_{i}}^{M} E_{i}\right)_{x}=0$ and $\sigma(x)=U_{\varphi(x)}=v$. From the Lemma 20 and theorem 7 we obtain:

$\tau(\sigma)_{x}=$ trace $_{g}(\nabla d \sigma)_{x}$ 


$$
\begin{aligned}
= & \sum_{i=1}^{m}\left\{\widetilde{\nabla}_{d \sigma\left(E_{i}\right)} d \sigma\left(E_{i}\right)\right\}_{(\varphi(x), v)} \\
= & \sum_{i=1}^{m}\left\{\widetilde{\nabla}_{\left[\left(d \varphi\left(E_{i}\right)\right)^{H}+\left(\nabla_{E_{i}}^{\varphi} \sigma\right)^{V}\right]}\left[\left(d \varphi\left(E_{i}\right)\right)^{H}+\left(\nabla_{E_{i}}^{\varphi} \sigma\right)^{V}\right]\right\}_{(\varphi(x), v)} \\
= & \sum_{i=1}^{m}\left\{\widetilde{\nabla}_{\left(d \varphi\left(E_{i}\right)\right)^{H}}\left(d \varphi\left(E_{i}\right)\right)^{H}+\widetilde{\nabla}_{\left(d \varphi\left(E_{i}\right)\right)^{H}}\left(\nabla_{E_{i}}^{\varphi} \sigma\right)^{V}+\widetilde{\nabla}_{\left(\nabla_{E_{i}}^{\varphi} \sigma\right)^{V}}\left(d \varphi\left(E_{i}\right)\right)^{H}\right. \\
& \left.+\widetilde{\nabla}_{\left(\nabla_{E_{i}}^{\varphi} \sigma\right)^{V}}\left(\nabla_{E_{i}}^{\varphi} \sigma\right)^{V}\right\}_{(\varphi(x), v)} \\
= & \sum_{i=1}^{m}\left[\left(\nabla_{d \varphi\left(E_{i}\right)}^{N} d \varphi\left(E_{i}\right)\right)^{H}-\frac{1}{2}\left(R^{N}\left(d \varphi\left(E_{i}\right), d \varphi\left(E_{i}\right)\right) \sigma\right)^{V}\right. \\
& +\left(\nabla_{d \varphi\left(E_{i}\right)}^{N} \nabla_{E_{i}}^{\varphi} \sigma\right)^{V}+\frac{\alpha}{2}\left(R^{N}\left(v, \nabla_{E_{i}}^{\varphi} \sigma\right) d \varphi\left(E_{i}\right)\right)^{H} \\
& +\frac{\alpha}{2}\left(R^{N}\left(v, \nabla_{E_{i}}^{\varphi} \sigma\right) d \varphi\left(E_{i}\right)\right)^{H} \\
& +\frac{\alpha^{\prime}}{\alpha}\left[h\left(\nabla_{E_{i}}^{\varphi} \sigma, v\right)\left(\nabla_{E_{i}}^{\varphi} \sigma\right)^{V}+h\left(\nabla_{E_{i}}^{\varphi} \sigma, v\right)\left(\nabla_{E_{i}}^{\varphi} \sigma\right)^{V}\right] \\
& \left.+\left(\frac{\beta-\alpha^{\prime}}{\alpha+r \beta} h\left(\nabla_{E_{i}}^{\varphi} \sigma, \nabla_{E_{i}}^{\varphi} \sigma\right)+\frac{\alpha \beta^{\prime}-2 \alpha^{\prime} \beta}{\alpha(\alpha+r \beta)} h\left(\nabla_{E_{i}}^{\varphi} \sigma, v\right)^{2}\right) U^{V}\right]_{(\varphi(x), v)}
\end{aligned}
$$

After offsetting the values of $r=h(\sigma, \sigma)=\|\sigma\|^{2}$ and $\sigma(x)=U_{\varphi(x)}=v$, we have:

$$
\begin{aligned}
\tau(\sigma)= & \sum_{i=1}^{m}\left[\left(\nabla_{E_{i}}^{\varphi} d \varphi\left(E_{i}\right)\right)^{H}+\alpha\left(R^{N}\left(\sigma, \nabla_{E_{i}}^{\varphi} \sigma\right) d \varphi\left(E_{i}\right)\right)^{H}\right. \\
& +\left(\nabla_{E_{i}}^{\varphi} \nabla_{E_{i}}^{\varphi} \sigma\right)^{V}+\frac{2 \alpha^{\prime}}{\alpha} h\left(\nabla_{E_{i}}^{\varphi} \sigma, \sigma\right)\left(\nabla_{E_{i}}^{\varphi} \sigma\right)^{V} \\
& \left.+\left(\frac{\beta-\alpha^{\prime}}{\alpha+\|\sigma\|^{2} \beta} h\left(\nabla_{E_{i}}^{\varphi} \sigma, \nabla_{E_{i}}^{\varphi} \sigma\right)+\frac{\alpha \beta^{\prime}-2 \alpha^{\prime} \beta}{\alpha\left(\alpha+\|\sigma\|^{2} \beta\right)} h\left(\nabla_{E_{i}}^{\varphi} \sigma, \sigma\right)^{2}\right) \sigma^{V}\right] \\
\tau(\sigma)= & {\left[\tau(\varphi)+\operatorname{trace}_{g}\left(\alpha R^{N}\left(\sigma, \nabla^{\varphi} \sigma\right) d \varphi(*)\right)\right]^{H} } \\
& +\left[\operatorname { t r a c e } _ { g } \left[\left(\nabla^{\varphi}\right)^{2} \sigma+\frac{2 \alpha^{\prime}}{\alpha} h\left(\nabla^{\varphi} \sigma, \sigma\right) \nabla^{\varphi} \sigma\right.\right. \\
& \left.\left.+\left(\frac{\beta-\alpha^{\prime}}{\alpha+\|\sigma\|^{2} \beta} h\left(\nabla_{E_{i}}^{\varphi} \sigma, \nabla_{E_{i}}^{\varphi} \sigma\right)+\frac{\alpha \beta^{\prime}-2 \alpha^{\prime} \beta}{\alpha\left(\alpha+\|\sigma\|^{2} \beta\right)} h\left(\nabla^{\varphi} \sigma, \sigma\right)^{2}\right) \sigma\right]\right]^{V}
\end{aligned}
$$

Theorem 22. Let $\left(M^{m}, g\right)$, $\left(N^{n}, h\right)$ two Riemannian manifolds and $f: N \rightarrow$ ] $0,+\infty\left[\right.$ a smooth function. Let $\left(T N, h^{f}\right)$ the tangent bundle of $N$ equipped with the Generalized Cheeger-Gromoll metric. The map

$$
\sigma:(M, g) \longrightarrow\left(T N, h^{f}\right)
$$




$$
x \longmapsto(\varphi(x), v)
$$

such that $\varphi=\pi_{T N} \circ \sigma$, is a harmonic if and only if the following conditions are verified

$$
\begin{aligned}
& \qquad(\varphi)=-\operatorname{trace}_{g}\left(\alpha R^{N}\left(\sigma, \nabla^{\varphi} \sigma\right) d \varphi(*)\right) \\
& \text { and } \\
& \qquad \begin{aligned}
0= & \operatorname{trace}_{g}\left[\left(\nabla^{\varphi}\right)^{2} \sigma+\frac{2 \alpha^{\prime}}{\alpha} h\left(\nabla^{\varphi} \sigma, \sigma\right) \nabla^{\varphi} \sigma\right. \\
& \left.+\left(\frac{\beta-\alpha^{\prime}}{\alpha+\|\sigma\|^{2} \beta} h\left(\nabla_{E_{i}}^{\varphi} \sigma, \nabla_{E_{i}}^{\varphi} \sigma\right)+\frac{\alpha \beta^{\prime}-2 \alpha^{\prime} \beta}{\alpha\left(\alpha+\|\sigma\|^{2} \beta\right)} h\left(\nabla^{\varphi} \sigma, \sigma\right)^{2}\right) \sigma\right]
\end{aligned}
\end{aligned}
$$

3.3. Harmonicity of the map $\phi:(T M, \widetilde{g}) \longrightarrow(N, h)$.

Lemma 23. Let $\left(M^{m}, g\right)$ be a Riemannian m-dimensional manifold and $(T M, \widetilde{g})$ its tangent bundle equipped with the Generalized Cheeger-Gromoll metric. The canonical projection

$$
\begin{aligned}
\pi:(T M, \widetilde{g}) & \longrightarrow(M, g) \\
(x, u) & \longmapsto x
\end{aligned}
$$

is harmonic: i.e. $\tau(\pi)=0$.

Proof. Let $(x, u) \in T M$ and $\left\{E_{i}\right\}_{i=\overline{1, m}}$ such that $E_{1}=\frac{u}{\|u\|}$ is an orthonormal basis on $M$ in $x$, then

$$
\left\{E_{i}^{H}, \frac{1}{\sqrt{\alpha+r \beta}} E_{1}^{V}, \frac{1}{\sqrt{\alpha}} E_{j}^{V}\right\}_{i=1, m}, j=\overline{2, m}
$$

is an orthonormal basis on $T M$ in $(x, u)$.

$$
\begin{aligned}
\tau(\pi)= & \operatorname{trace}_{\widetilde{g}} \nabla d \pi \\
= & \sum_{i=1}^{m}\left\{\nabla_{E_{i}^{H}}^{\pi} d \pi\left(E_{i}^{H}\right)-d \pi\left(\nabla_{E_{i}^{H}}^{T M} E_{i}^{H}\right)\right\} \\
& +\nabla_{\left(\frac{1}{\sqrt{\alpha+r \beta}} E_{1}^{V}\right)}^{\pi} d \pi\left(\frac{1}{\sqrt{\alpha+r \beta}} E_{1}^{V}\right)-d \pi\left(\nabla_{\left(\frac{1}{\sqrt{\alpha+r \beta}} E_{1}^{V}\right)}^{T M}\left(\frac{1}{\sqrt{\alpha+r \beta}} E_{1}^{V}\right)\right) \\
& +\sum_{j=2}^{m}\left\{\nabla_{\left(\frac{1}{\sqrt{\alpha}} E_{j}^{V}\right)}^{\pi} d \pi\left(\frac{1}{\sqrt{\alpha}} E_{j}^{V}\right)-d \pi\left(\nabla_{\left(\frac{1}{\sqrt{\alpha}} E_{j}^{V}\right)}^{T M}\left(\frac{1}{\sqrt{\alpha}} E_{j}^{V}\right)\right)\right\}
\end{aligned}
$$

as $d \pi\left(E_{i}^{V}\right)=0$ and $d \pi\left(E_{i}^{H}\right)=E_{i} \circ \pi$ then:

$$
\begin{aligned}
\tau(\pi)= & \sum_{i=1}^{m}\left\{\left(\nabla_{E_{i}}^{M} E_{i}\right) \circ \pi-d \pi\left(\nabla_{E_{i}}^{M} E_{i}\right)^{H}\right\} \\
& -\frac{1}{\sqrt{\alpha+r \beta}} d \pi\left[E_{1}^{V}\left(\frac{1}{\sqrt{\alpha+r \beta}}\right) E_{1}^{V}+\frac{1}{\sqrt{\alpha+r \beta}} \nabla_{E_{1}^{V}}^{T M} E_{1}^{V}\right]
\end{aligned}
$$




$$
\begin{aligned}
& -\sum_{j=2}^{m}\left\{\frac{1}{\sqrt{\alpha}} d \pi\left[E_{j}^{V}\left(\frac{1}{\sqrt{\alpha}}\right) E_{j}^{V}+\frac{1}{\sqrt{\alpha}} \nabla_{E_{j}^{V}}^{T M} E_{j}^{V}\right]\right\} \\
= & -\frac{1}{\alpha+r \beta} d \pi\left(\nabla_{E_{1}^{V}}^{T M} E_{1}^{V}\right)-\sum_{j=2}^{m}\left\{\frac{1}{\alpha} d \pi\left(\nabla_{E_{j}^{V}}^{T M} E_{j}^{V}\right)\right\} \\
= & 0
\end{aligned}
$$

Theorem 24. Let $\left(M^{m}, g\right)$, $\left(N^{n}, h\right)$ two Riemannian manifolds and $f: M \rightarrow$ ] $0,+\infty[$ a smooth function. Let $(T M, \widetilde{g})$ the tangent bundle of $M$ equipped with the Generalized Cheeger-Gromoll metric.

Let $\varphi:(M, g) \longrightarrow(N, h)$ a smooth map. The tension field of the map:

$$
\begin{aligned}
\phi:(T M, \widetilde{g}) & \longrightarrow(N, h) \\
(x, y) & \longmapsto \varphi(x)
\end{aligned}
$$

is given by:

$$
\tau(\phi)=[\tau(\varphi)] \circ \pi
$$

Proof. Let $(x, u) \in T M$ and $\left\{E_{i}\right\}_{i=\overline{1, m}}$ such that $E_{1}=\frac{u}{\|u\|}$ is an orthonormal basis on $M$ in $x$, then $\left\{E_{i}^{H}, \frac{1}{\sqrt{\alpha+r \beta}} E_{1}^{V}, \frac{1}{\sqrt{\alpha}} E_{j}^{V}\right\}_{i=\overline{1, m}, j=\overline{2, m}}$ is an orthonormal basis on $T M$ in $(x, u)$.

as the $\phi$ is defined by:

$$
\begin{aligned}
\phi:(T M, \widetilde{g}) & \stackrel{\pi}{\longrightarrow}(M, g) \stackrel{\varphi}{\longrightarrow}(N, h) \\
(x, y) & \longmapsto x \longmapsto \varphi(x)
\end{aligned}
$$

i.e. $\phi=\varphi \circ \pi$, we have:

$$
\begin{gathered}
\tau(\phi)=\tau(\varphi \circ \pi) \\
=d \varphi(\tau(\pi))+\operatorname{trace}_{\widetilde{g}} \nabla d \varphi(d \pi, d \pi) \\
\operatorname{trace}_{\tilde{g}} \nabla d \varphi(d \pi, d \pi)=\sum_{i=1}^{m}\left\{\nabla_{d \pi\left(E_{i}^{H}\right)}^{\varphi} d \varphi\left(d \pi\left(E_{i}^{H}\right)\right)-d \varphi\left(\nabla_{d \pi\left(E_{i}^{H}\right)}^{M} d \pi\left(E_{i}^{H}\right)\right)\right\} \\
+\sum_{j=2}^{m}\left\{\nabla_{d \pi\left(\frac{1}{\sqrt{\alpha}} E_{j}^{V}\right)}^{\varphi} d \varphi\left(d \pi\left(\frac{1}{\sqrt{\alpha}} E_{j}^{V}\right)\right)-d \varphi\left(\nabla_{d \pi\left(\frac{1}{\sqrt{\alpha}} E_{j}^{V}\right)}^{M} d \pi\left(\frac{1}{\sqrt{\alpha}} E_{j}^{V}\right)\right)\right\} \\
+\nabla_{d \pi\left(\frac{1}{\sqrt{\alpha+r \beta}} E_{1}^{V}\right)}^{\varphi} d \varphi\left(d \pi\left(\frac{1}{\sqrt{\alpha+r \beta}} E_{1}^{V}\right)\right)-d \varphi\left(\nabla_{d \pi\left(\frac{1}{\sqrt{\alpha+r \beta}} E_{1}^{V}\right)}^{M} d \pi\left(\frac{1}{\sqrt{\alpha+r \beta}} E_{1}^{V}\right)\right) \\
=\sum_{i=1}^{m}\left\{\left(\nabla_{E_{i}}^{\varphi} d \varphi\left(E_{i}\right)\right) \circ \pi-d \varphi\left(\nabla_{E_{i}}^{M} E_{i}\right) \circ \pi\right\} \\
=\sum_{i=1}^{m}\left\{\nabla_{E_{i}}^{\varphi} d \varphi\left(E_{i}\right)-d \varphi\left(\nabla_{E_{i}}^{M} E_{i}\right)\right\} \circ \pi
\end{gathered}
$$




$$
=\tau(\varphi) \circ \pi
$$

Using Lemma 23, we obtain:

$$
\tau(\phi)=\tau(\varphi) \circ \pi
$$

Theorem 25. Let $\left(M^{m}, g\right),\left(N^{n}, h\right)$ two Riemannian manifolds and $f: M \rightarrow$ ] $0,+\infty[$ a smooth function. Let $(T M, \widetilde{g})$ the tangent bundle of $M$ equipped with the Generalized Cheeger-Gromoll metric. The map

$$
\begin{aligned}
\phi:(T M, \widetilde{g}) & \longrightarrow(N, h) \\
(x, y) & \longmapsto \varphi(x)
\end{aligned}
$$

is harmonic if and only if $\varphi$ is a harmonic.

Acknowledgement. The authors would like to thank the reviewers for their useful remarks and suggestions. Partially supported by PRFU National Agency Scientific Research of Algeria.

\section{REFERENCES}

[1] Abbassi, M.T.K. and Sarih, M., On natural metrics on tangent bundles of Riemannian manifolds, Arch. Math., 41 (2005), 71-92.

[2] Anastasiei, M., Locally conformal Kaehler structures on tangent bundle of a space form, Libertas Math., 19 (1999), 71-76.

[3] Boeckx, E. and Vanhecke, L., Harmonic and minimal vector fields on unit tangent bundles, Differential Geometry and Applications, Volume 13, Issue 1, July 2000, Pages 77-93.

[4] Calvaruso, G., Naturally Harmonic Vector Fields, Note di Matematica, Note Mat., suppl.n.1, 1 (2008), 107-130.

[5] Cheeger, J. and Gromoll, D., On the structure of complete manifolds of nonnegative curvature, Ann. of Math., (2) 96 (1972), 413-443.

[6] Cengiz, N., Salimov, A.A., Diagonal lift in the tensor bundle and its applications. Appl. Math. Comput. 142, no.2-3 (2003), 309-319.

[7] Cherif, A.M. and Djaa, M., On the Biharmonic maps with potential, Arab Journal Mathemaical Sciences, AJMS Elsevier, 24(1) (2018), 1-8.

[8] Djaa, M. and Cherif, A. M., On Generalized $f$-biharmonic Maps and Stress $f$-bienergy Tensor, Journal of Geometry and Symmetry in Physics JGSP, 29 (2013), pp. 65-81.

[9] Djaa, M., Mohamed Cherif, A., Zegga, K. And Ouakkas, S., On the Generalized of harmonic and Bi-harmonic Maps, International electronic journal of geometry, 5 no. 1 (2012), 90-100.

[10] Djaa, M. and Gancarzewicz, J., The geometry of tangent bundles of order $r$, Boletin Academia , Galega de Ciencias, Espagne, 4 (1985), 147-165

[11] Djaa, N.E.H., Ouakkas, S. and Djaa, M., Harmonic sections on the tangent bundle of order two, Annales Mathematicae et Informaticae, 38 (2011), 15-25.

[12] Djaa, N.E.H., Boulal, A. and Zagane, A., Generalized warped product manifolds and Biharmonic maps, Acta Math. Univ. Comenianae, Vol. LXXXI, 2 (2012), 283-298.

[13] Dombrowski, P., On the geometry of tangent bundle, J. Reine Angew .Math., 210 (1962), 73-88.

[14] Ells, J. and Sampson, J.H., Harmonic mappings of Riemannian manifolds. Amer.J. Maths., 86 (1964).

[15] Ells, J. and Lemaire, L., Another report on harmonic maps, Bull. London Math. Soc., 20 (1988), 385-524. 
[16] Gezer, A. and Altunbas, M., Some notes concerning Riemannian metrics of Cheeger-Gromoll type, J. Math. Anal. Appl., 396 (2012) 119-132.

[17] Gudmunsson, S. and Kappos, E., On the Geometry of Tangent Bundles, Expo.Math., 20 (2002),1-41.

[18] Ishihara, T., Harmonic sections of tangent bundles, J. Math. Tokushima Univ., 13 1979), 23-27.

[19] Konderak, J.J., On Harmonic Vector Fields, Publications Mathematiques, Vol 36, (1992), 217-288.

[20] Latti, F., Djaa, M. and Zagane, A., Mus-Sasaki Metric and Harmonicity, Mathematical Sciences and Applications E-Notes, 6 (1) (2018), 29-36.

[21] Munteanu, M., Some Aspects on the Geometry of the Tangent Bundles and Tangent Sphere Bundles of Riemannian Manifold, Mediterr. J. Math., 5 (2008), 43-59.

[22] Opriou, V., On Harmonic Maps Between tangent bundles, Rend. Sem.Mat., Vol 47, 1(1989).

[23] Salimov, A. A., Gezer, A. and Akbulut, K., Geodesics of Sasakian metrics on tensor bundles, Mediterr. J. Math., 6, no.2 (2009), 135-147.

[24] Salimov, A. A. and Kazimova, S., Geodesics of the Cheeger-Gromoll Metric, Turk J Math., 33 (2009), $99-105$.

[25] Sasaki, S., On the differential geometry of tangent bundles of Riemannian manifolds, Tohoku Math. J., 10 (1958), 338-354.

[26] Sekizawa, M., Curvatures of Tangent Bundles with Cheeger-Gromoll Metric, Tokyo J. Math., 14, No. 2 (1991), 407-417.

[27] Yano, K. and Ishihara, S., Tangent and Cotangent Bundles, Marcel Dekker INC. New York, 1-171, 1973.

[28] Zagane, A. and Djaa, M., On Geodesics of Warped Sasaki Metric, Mathematical Sciences and Applications E-Notes 5 (1) (2017), 85-92.

Current address: R. KADA Ben OTMANE: Mascara University, Dept.of Mathematics, 29000, Mascara-Algeria.

E-mail address: rida.kadabenothmane@univ-mascara.dz

ORCID Address: http://orcid.org/0000-0002-0185-6324

Current address: Aberrahim Zagane: University of Oran1 Ahmed Ben Bella, Dept. of Mathematics, 31000, Oran-Algeria.

E-mail address: Zagane2016@gmail.com

ORCID Address: http://orcid.org/0000-0001-9339-3787

Current address: Mustapha Djaa: Relizane University, Dept.of Mathematics,48000, RelizaneAlgeria.

E-mail address: Djaamustapha@live.com

ORCID Address: http://orcid.org/0000-0002-7330-2144 